\title{
New Cancer Therapies: Implications for the Perioperative Period
}

\author{
Tayab Andrabi ${ }^{1}$ - Katy E. French ${ }^{2}$ - Muzaffar H. Qazilbash ${ }^{3}$ \\ Published online: 6 December 2018 \\ (C) Springer Science+Business Media, LLC, part of Springer Nature 2018
}

\begin{abstract}
Purpose of Review Cancer is on the rise. Standing on verge of exciting discoveries, research is being translated into therapies that are being widely administered to patients. Providing a hope for cure, where none existed before. This new body of knowledge has come from a better understanding of cancer genetics, molecular and sub molecular behavior, and understanding of cancergenerated cellular environments. These have led to development of immunotherapy and its many sub-genres, improvement and introduction of new radiation technologies, and decreasing toxicities of existing chemotherapies.

Recent Findings The purpose of this review is to have a summary look at this huge landscape of cancer therapy. Specially looking at toxicities that an anesthesiologist should be familiar with while providing perioperative care for these patients, complications like tumor lysis syndrome, cytokine release syndromes, Kounis syndrome, myocarditis, encephalopathies, and pituitary failure need to be kept in mind.

Summary One should be knowledgeable about these therapies and approach these patients with a high index of suspicion. Anesthesiologists will need to refine preoperative assessment with appropriate testing and intraoperative and postoperative management in collaboration with oncologists, while involving the expertise of internists, cardiologist, and endocrinologists in helping assess and manage these patients in the perioperative period.
\end{abstract}

Keywords Anesthesia assessment in cancer patients $\cdot$ Perioperative implications of cancer therapy $\cdot$ New cancer therapies, side effects, and management in perioperative period $\cdot$ Immunotherapy, mechanism of actions, and side effects $\cdot$ Side effects

This article is part of the Topical Collection on Cancer Anesthesia

Tayab Andrabi

tandrabi@mdanderson.org

Katy E. French

kefrench@mdanderson.org

Muzaffar H. Qazilbash

mqazilba@mdanderson.org

1 Anesthesiology and PeriOper Med, Margaret and Ben Love Clinic (R6.1365), The University of Texas MD Anderson Cancer Center, 1515 Holcombe Blvd.Unit 0409, Houston, TX 77030, USA

2 Anesthesiology and PeriOper Med, Margaret and Ben Love Clinic (R6.1370), The University of Texas MD Anderson Cancer Center, 1515 Holcombe Blvd. Unit 001, Houston, TX 77030, USA

3 Stem Cell Transplantation, John Mendelsohn Faculty Center (FC5.2006), The University of Texas MD Anderson Cancer Center, 1515 Holcombe Blvd. Unit 0423, Houston, TX 77030, USA

\section{Introduction}

In 2012, there were an estimated 14.1 million cases of cancer diagnosed around the world and 8.2 million cancer deaths. By 2030, the global burden is expected to reach 21.6 million new cancer cases and 13.0 million cancer deaths (American Cancer Society. Cancer Facts \& Figures 2018. Atlanta: American Cancer Society; 2018). Research has flourished not only in the traditional areas of prevention, early detection, diagnosis, and treatment, but newer insights into cancer biology and genomics have led to the development of many novel therapies. While these treatments hold new promises changing the landscape of cancer therapy, they do come with side effects and unique toxicities. (www.cancer.gov).

Cancer treatments can be broadly categorized into the following:

1. Surgery

2. Chemotherapy

3. Radiation 
a) External Beam

b) Internal (Brachytherapy or localized radiation modalities)

4. Hormone Therapy

5. Immunotherapy

6. Targeted Therapy

\section{Chemotherapy}

The classical chemotherapeutic agents generally target fast dividing cancer cell but also injure normal cells. Chemotherapy is termed as neoadjuvant when given before surgery, adjuvant when given during or after surgery, and palliative when given to improve the quality of life. Toxicities affect pulmonary, cardiac, renal, hepatic, gastrointestinal, and hematologic and nervous systems [1], illustrated by the following examples:

Bleomycin, an antitumor antibiotic isolated from a strain of Streptomyces verticillus. It is used for treating Hodgkin's lymphoma, cervical cancers, and germ-cell tumors. It is known to cause pulmonary injury leading to pulmonary fibrosis. Pulmonary toxicity is accelerated with exposure to highinspired concentration of oxygen. Bleomycin-induced lung injury or BILI is from release of cytokines and free radicals, related to low levels of bleomycin hydrolase (BLMH) in the lung. BLMH deactivates bleomycin. It is believed that in the presence of higher inspired oxygen concentrations, free radical formation increases, accelerating lung injury. This leads to an immunologically mediated inflammatory response causing a pneumonitis, eventually culminating in pulmonary fibrosis [2]. Bleomycin-induced pulmonary toxicity is seen in about $40-45 \%$ of patients with a mortality reaching $3 \%$. Risk factors include age, smoking, cumulative drug dose, poor renal function, concomitant use of oxygen, and radiation therapy.

There are reports of cases developing acute respiratory distress syndrome ARDS after surgeries requiring general anesthesia, interestingly a retrospective analysis found the incidence at $1.9 \%$, lower than expected.[3••]

Therapy for bleomycin-induced lung toxicity has not been established. There is role of corticosteroids in acute phases of interstitial pneumonitis and hypersensitivity pneumonitis (prednisone up to $100 \mathrm{mg} /$ day).

Supplemental oxygen should be used to keep oxygen saturations at $89-92 \%$; in practice, anesthesiologist will tend to keep the $\mathrm{FiO} 2$ to no more than $30 \%$. The priority remains to maintain adequate oxygen saturations even if higher inspired $\mathrm{O} 2$ concentrations are needed. Intraoperatively judicious use of intravenous fluids to avoid pulmonary edema is recommended [4].

Epirubicin and doxorubicin are anthracyclines commonly used for treating breast cancer. Cardiotoxic complications range from benign arrhythmias to ischemia/myocardial infarction, heart failure, and cardiomyopathy with reduced ejection fraction [5]. In most cases, toxicity manifests in the first year after chemotherapy and depends on total anthracycline dose and posttreatment LVEF [6]. Interestingly, in some animal studies, melatonin use has shown to be protective against adriamycin-induced toxicity [7]. Echocardiogram is still the mainstay of diagnosing the condition, but increasingly biomarkers like BNP and troponin fractions are being utilized. However, early detection and prompt treatment are crucial for regaining ventricular function, which seems to recover in majority of cases [6].

5-fluro-uracil (5-FU) another drug used in breast and colon cancers can injure coronary arteries producing angina-like syndrome and even myocardial infarctions. Cisplatin, carboplatin, and oxaliplatin, platinum-based chemotherapy agents are nephrotoxic.

Cisplatin and vincristine can also cause neurotoxicity-like, peripheral, and cranial neuropathies, myalgia, and seizures [8•]. Most chemo agents are used as combinations effecting multiple systems myelo-suppression, anemia, neutropenia, and pancytopenia. Gastro-intestinal side effects like nausea, vomiting, diarrhea, and mucositis are common and often debilitating [9].

\section{Radiation}

Radiation therapy uses high-energy particles or waves, such as $\mathrm{x}$-rays, gamma rays, electron beams, or protons, to destroy or damage cancer cells, by creating free-oxygen radicals leading to breaks in nuclear DNA. Radiation can be given as external radiation, internal radiation or brachytherapy, and systemic radiation (given as an oral radioactive drug that circulates systemically but eventually concentrates in the target tumor cell, e.g., radioactive iodine for thyroid tumors).

\section{External Beam Radiation or Photon Beam Radiation Therapy} Photons are charged through a linear accelerator delivering a photon beam to the tumor. This can damage healthy tissue in the path of the beam and around the tumor. Depending on the site of radiation, significant damage can occur causing delayed wound healing, induration of the skin, vascular stenosis, myocarditis, pneumonitis, pulmonary fibrosis, and delayed wound healing. Radiation for head and neck cancers leads to induration, fibrosis, skin changes, and mucositis. Achieving a central venous access is difficult; face mask ventilation and laryngoscopy become challenging due to limited neck extension and rigidity of the oropharyngeal tissues. Radiotherapy for the head and neck leads to a difficult central venous access.

Over time newer radiation techniques have developed to (a) deliver precise high doses to tumor cells and (b) minimize damage to surrounding healthy tissue. 
Proton Beam Radiation Therapy A cyclotron or synchrotron generates a proton beam uses protons, causing little damage to tissues they pass through. The cell killing energy is released at a calculated depth (tumor site). A proton beam radiation delivers more radiation to the tumor while reducing damage to normal tissues.

Three-dimensional conformal radiation therapy (3D-CRT): A 3-d image of the tumor is constructed and then radiation is delivered from different angles matching tumor shape, reducing normal tissue damage [10].

Image-guided radiation therapy (IGRT) is like 3D-CRT. Radiation is adjusted to hit the tumor precisely based on CT images. Intensity-modulated radiation therapy (IMRT) and helical-tomotherapy are other variations of 3D-CRT $[11,12]$.

Stereotactic radiosurgery is used primarily for brain tumors. Radiation precisely aimed at the tumor, giving a large dose of radiation to a small tumor area, minimizing collateral damage [13]. When used outside the brain, it is termed stereotactic body radiation therapy (SBRT), used for certain lung, spine, and liver tumors. Examples are X-Knife ${ }^{\mathrm{TM}}$, CyberKnife ${ }^{\circ}$, Clinac ${ }^{\circledR}$, and Gamma Knife.

Intraoperative radiation therapy (IORT) is external radiation given directly to the tumor or tumors during surgery [14].

\section{Hormone Therapy}

Some cancers require hormones to grow. Therapy is directed towards stopping hormone production or preventing their growth effects on cancer cells. Breast, prostate, ovarian, and endometrial cancers can be hormone sensitive. Drugs like tamoxifen, aromatase inhibitors like anastrazole (Arimidex), exemestane (Aromasin), letrozole (Femara), and leutinizing hormone blocker gosserlin (Zoladex) have been used in breast cancers. Side effects include propensity for forming blood clots, liver impairment, and blood pressure changes. Similarly for prostate cancers, anti-androgen drugs like enzalutamide (Xtandi) and abiraterone (Zytiga) are being used. About $10 \%$ report hypertension as a side effect and cardiac dysrythmias are reported about $1 \%$ of the time. This is attributed to mineralocorticoid excess. Hepatic impairment has also been reported. (www.medicines.org.uk).

\section{Targeted Therapy}

Targeted therapy refers to a new generation of cancer drugs designed to interfere with a specific molecular target (typically a protein) that is believed to have a critical role in tumor growth or progression. There are two main groups: (1) small molecule inhibitors and (2) angiogenesis inhibitors. U.S. Food and Drug Administration (FDA) has approved over 15 targeted cancer therapies since 2000 [15•].

Small Molecule Inhibitors This group targets proteins that have become dysregulated, such as inappropriately overactive kinase activity rendering a cell cancerous. By binding to tyrosine kinase site, these molecules inactivate the domain, thus disrupting downstream signaling. General use is to treat chronic myeloid leukemia (CML) and gastro-intestinal stromal tumors (GIST). The two commonly used drugs in this category are imatinib (Gleevec) and dasatinib (Sprycel). Imatinib targets abelson tyrosine kinase (ABL) protein, which also modulates function in cardiac muscle cells, leading to left ventricular dysfunction and heart failure (in less than $1 \%$ of patients). Common side effects include an increase in infection rates, diarrhea, headaches, pleural effusions, and myalgias [16].

Angiogenesis Inhibitors Angiogenesis means formation of new blood vessels. Along with proliferation of endothelium, this plays a key role in tumor growth. Thus, blocking angiogenesis is a logical approach against many tumor type. Signaling systems that regulate this process are vascular endothelium growth factor (VEGF) and their receptors (VEGFR-1, -2, and -3). VEGFR-2). VEGFRs are expressed at high levels in many types of human solid tumors, including glioma, lung, breast, renal, ovarian, and gastrointestinal tract carcinomas. Inhibition of VEGFR has led to development of drugs like bevacizumab, sorafenib, and suntinib [17]. Side effects include HTN (can progress to hypertensive encephalopathy in some cases), dysrhythmias, myocardial dysfunction, (QTc prolongation) strokes, and rarely, a condition called reversible posterior leukoencephalopathy (RPLNS) that manifests as eyesight changes, drowsiness, and behavioral changes. Skin toxicities like hand-foot syndrome (palmo-plantar erythrodysesthesia), xerosis, exanthens, and scaling are common. In a surgical patient, these can lead to delayed wound healing, but these side effects are dose-related and reversible with discontinuation of therapy; thus, a cooling off period of 4-6 weeks generally was observed prior to surgery $[18,19]$.

Formation of blood vessels maybe a useful way to arrest tumor growth; however, it has the opposite effect on wound healing. Angiogenesis is critical to proliferative phase of wound healing. Inhibition of VEGF can therefore produce surgical site bleeds, wound dehiscence, wound infections, and incisional hernias [20••].

\section{Immunotherapy}

Spontaneous regression of a tumor is an extremely rare ( 1 in $60,000-100,000$ ) event. Immune-mediated host responses are thought to be central to this phenomenon [21, 22]. Cancer 
immunotherapy is now a rapidly expanding field and encompasses a wide variety of concepts and methods. There are more than 3000 immuno-oncology trials ongoing, targeting hundreds of disease and immune pathways and involving hundreds of research institutes across the globe. Increased understanding of the complex interactions between tumors and the host immune response has led to the development of newer therapeutic strategies. In particular, a wide range of different and potentially synergistic immunotherapy combinations $[23 \bullet \bullet]$.

Non-specific Cancer Immunotherapies This type of therapy does not target cancer cells directly, but stimulates the immune system in a general way to improve the immune response. These can be used alone, or as adjuvants. Grouped under cytokines, interferons, interleukins, immune-modulating drugs like thalidomide and lenalidomide. These have now been in use for many years. Side effects include fatigue, constipation, and low blood cell count. More serious side effects are neuropathies and DVT/PE.

Monoclonal Antibodies In 1975, development of hybridoma technology enabled the production of mouse monoclonal antibodies (mAbs) [24]. Since then, transition from mouse to fully human mAbs has successfully taken place leading to successful clinical use. Monoclonal antibodies are designed to identify and attach themselves to surface antigens on tumor cells thus rendering them visible to host immune system (alemtuzumab (Campath)) leading to direct inhibition of tumor cell proliferation by apoptosis or inhibiting cell growth by inactivating key proteins, (trastuzumab (Herceptin)) or kill targeted tumor cells through antibody-dependent and complement-dependent cell-mediated cytotoxicity (ADCC and CDC). Antibodies can recruit immune cells such as T cells or chimeric antigen receptor (CAR) $\mathrm{T}$ cells and can also be used to deliver chemo (brentuximab (Adcetris)) and radiation (ibritumomabtiuxetan (Zevalin) to cancer cells [25]. As expected, a multitude of general immune-mediated side effects like allergic reactions (including anaphylaxis), serum sickness, tumor lysis syndrome, thrombocytopenia, anemia, and dermatitis are common. More serious side effects like cytokine release syndrome, re-activation of latent tuberculosis, progressive multifocal leuco-encephalopathy, generally due to reactivation of John Cunningham virus, are often fatal [26].

In 1991, Kounis et al. described histamine-induced coronary artery spasm or allergic angina syndrome [27]. This is a cardiac hypersensitivity reaction due to the formation of antimonoclonal drug antibodies, mediated through mast cell activation. Coronary artery spasm and atheromatous plaque rupture occur during an allergic reaction. Three variants are recognized: vaso-spastic allergic angina, allergic myocardial infarction, and stent thrombosis leading to ventricular dysfunction, arrhythmias, acute myocardial infarction, and vasospastic angina pectoris [28]. Diagnosis is based on symptoms of chest pain, ST-elevation and acute MI on EKG, elevated troponins, and wall motion abnormalities on echocardiogram. Treatment includes the use of antihistamines, steroids, and sometimes percutaneous coronary interventions.

Hyperthyroidism, responsive to carbimazole, has been reported [29, 30].

Immune Checkpoint Inhibitors Immune response to a pathogen is a complex, multimodal response with built-in checks and balances to limit collateral damage to innocent bystanders. The molecules that suppress the immune response when it encounters a normal cell are called immune checkpoints; so, under normal physiologic conditions, the immune checkpoints serve to inhibit immune responses against selfantigens, thereby preventing unwanted autoimmunity [31]. Tumor cells often utilize immune checkpoint molecules to avoid $\mathrm{T}$ cell recognition and thus evade an immune attack. PD-1 and PD L-1 are checkpoint proteins that can be blocked to achieve checkpoint inhibition. Pembolizumab (Keyturda), nivolumab (Opdivo), atezolizumab (Tecentriq), avelumab (Bavencio), and durvulumab (Imfinzi) are currently approved for use in non-small cell lung cancers and melanomas.

CTLA-4 is another check point protein, and ipilimumab (Yervoy), a monoclonal antibody, used against melanoma, but has a more serious side effect profile.

Newer check point inhibitors, e.g., tremelimumab, pidilizumab, and eluzumab are in the pipeline [32].

With these therapies, the breaking up of immune selftolerance leads to a myriad of auto-immune reactions. These range from appearance of rashes and GI dysfunction like colitis and hepatitis to life-threatening endocrinopathies. Ipilimumab treatment has been associated with hypothyroidism, adrenal insufficiency, and hypophysitis. Hyopophysitis can be life-threatening due to secondary hypoadrenalism. It is irreversible in most cases requiring lifelong hormone replacement [33].

Adoptive Cell Therapy Cancer cells are remarkably adept in escaping immune surveillance. Patients' own T cells can be modified by adding an antigen receptor to the cell in the lab, thus forming a chimeric antigen receptor (CAR) T lymphocyte. Patients' T cells are removed by leukopharesis, modified, and re-infused. Tisagenlecleucel (Kymryeah) has recently been approved by FDA to be used in B-cell type acute lymphocytic leukemia. The therapy is promising but has some serious side effects like CNS toxicities and cytokine release syndrome (CRS), which occur as cancer cells lyse and release toxins in the blood, further stimulating the immune system to unleash a massive response. CRS is a potentially lifethreatening toxicity mediated through cytokines like interferon y, and interleukin (IL) 6. Symptoms range from fever, headache, chills, fatigue, and diarrhea to dysrhythmias and 
heart failure. Drugs like tocilizumab (an immunosuppressant) and corticosteroids are effective treatments, but this then defeats the purpose of immunotherapy [34].

Therapeutic tumor-infiltrating lymphocytes (TILs) is another form of adoptive cell therapy that [35] employs isolating lymphocytes from the tumor tissue, modifying these in vitro by treating them with IL-2 and re-infused in the tumor leading to tumor lysis [36]. Treatment is being tested on melanoma and the liver and kidney cancers. Rare but serious side effects include cytokine-release syndrome, cardiac dysrhythmias, shock, and coma $[37 \bullet \bullet]$.

Vaccines Bacillus Calmette-Guerrin (BCG) has long been used to treat urinary bladder cancers. It is instilled into the bladder few weeks after a TURBT. The BCG attaches to cancer cells. Circulating immune cells are attracted to the bacillus, coming in close contact with superficial surface lining cancer cells, attacking and killing them. HPV (human papillomavirus) and hepatitis B vaccines have also been used for the same purpose.

Tumor cell, tumor antigen, and vector-based vaccines are under study. Dendritic cell vaccines have shown the most success, and sipulecel (Provenge) has been approved for use in prostate cancer.

Oncolytic viruses are being studied to kill cancer cells or immune, modify, and boost the immune system to attack cancer cells. Talimogene laherparepvec (Imlygic) is one such example [35].

\section{Conclusion}

It is hard for an anesthesiologist to keep pace with all the new therapeutic developments that have come about in a short period of time. At the same time, patients who receive these treatments are not only physiologically compromised but may come in with specific side effects from treatment that the anesthesiologist needs to be fully aware of. This will require a good working knowledge of abovementioned treatment modalities, a thorough preoperative assessment, communication with oncologists and surgeons, but most importantly extreme vigilance during anesthesia.

The cancer state poses a unique challenge when one ventures to determine the functional status of a patient from a preoperative assessment standpoint. The purpose of this assessment among other things is to clarify patient and surgical specific risks and recognize when such a risk can be optimized in a patients favor. Cancer-related fatigue and treatmentinduced physiological derangements make it difficult to identify where the source of physiological dysfunction lies. It may be impossible to assess the integrity of the cardiovascular system based on physical activity (a key determinant of cardiac integrity in ACC/AHA guidelines). When on top of this, a patient has undergone therapies that are cardio toxic, the picture becomes fuzzier. One then has to rely on investigations like serial troponins, echocardiograms, and their interpretations to determine as true a cardiac status as possible. If cardiac injury is found, then one must determine the necessity vs risk of surgery and the effects of delaying cancer therapy. If we do need to "cancel" a case, we must ensure that the time gained by this delay is primarily used to reduce the assessed risk that has resulted in the delay. These decisions have to be made in conjunction with the patient, surgeon, and oncologists involved in patient care. The dialog should be transparent with clearly established goals. The need for collaboration, advice, and consent with colleagues from surgery, medicine, cardiology, and oncology has now become more important than ever before.

\section{Compliance with Ethical Standards}

Conflict of Interest TayabAndrabi, Katy E. French, and Muzaffar H. Qazilbash declare they have no conflict of interest.

Human and Animal Rights and Informed Consent This article does not contain any studies with human or animal subjects performed by any of the authors.

\section{References}

Papers of particular interest, published recently, have been highlighted as:

- Of importance

•- Of major importance

1. Huitink JM, Teoh WH. Current cancer therapies - a guide for perioperative physicians. Best Pract Res Clin Anaesthesiol. 2013;27(4): 481-92.

2. Sleijfer S. Bleomycin-induced pneumonitis. Chest. 2001;120(2): 617-24.

3.• Aakre BM, Efem RI, Wilson GA, Kor DJ, Eisenach JH. Postoperative acute respiratory distress syndrome in patients with previous exposure to bleomycin. Mayo Clin Proc. 2014;89(2): 181-9 Risk of postoperative ARDS in patients exposed to systemicbleomycin appears to be lower than expected. Smoking status may be an important factor that modifies the risk of postoperative ARDS.

4. Goldiner PL, Carlon GC, Cvitkovic E, Schweizer O, Howland WS. Factors influencing postoperative morbidity and mortality in patients treated with bleomycin. Br Med J. 1978;1(6128):1664-7.

5. Gudaityte J, Dvylys D, Simeliunaite I. Anaesthetic challenges in cancer patients: current therapies and pain management. Acta Med Austriaca. 2017;24(2):121-7.

6. Cardinale D, Colombo A, Bacchiani G, Tedeschi I, Meroni CA, Veglia F, et al. Early detection of anthracycline cardiotoxicity and improvement with heart failure therapy. Circulation. 2015;131(22): 1981-8.

7. Bilginoglu A, Aydin D, Ozsoy S, Aygun H. Protective effect of melatonin on adriamycin-induced cardiotoxicity in rats. Turk 
Kardiyoloji Dernegi arsivi: Turk Kardiyoloji Derneginin yayin organidir. 2014;42(3):265-73.

8. Sahai SK. Perioperative assessment of the cancer patient. Best Pract Res Clin Anaesthesiol. 2013;27(4):465-80 This article reviews the perioperative concerns that are specific to thepatient with cancer.

9. Riechelmann RP, Tannock IF, Wang L, Saad ED, Taback NA, Krzyzanowska MK. Potential drug interactions and duplicate prescriptions among cancer patients. J Natl Cancer Inst. 2007;99(8): 592-600.

10. Zou L-Q, Zhang B-L, Chang Q, Zhu FP, Li YY, Wei YQ, et al. 3D conformal radiotherapy combined with transcatheter arterial chemoembolization for hepatocellular carcinoma. World $\mathrm{J}$ Gastroenterol: WJG. 2014;20(45):17227-34.

11. Zelefsky MJ, Kollmeier M, Cox B, et al. Improved clinical outcomes with high-dose image guided radiotherapy compared with non-IGRT for the treatment of clinically localized prostate cancer. Int J Radiat Oncol Biol Physics. 2012;84(1):125-9.

12. Fu J, Willner M, Chen L, Tan R, Achterhold K, Bech M, et al. Helical differential X-ray phase-contrast computed tomography. Physica Medica. 2014;30(3):374-9.

13. Yamamoto M, Serizawa T, Shuto T, Akabane A, Higuchi Y, Kawagishi J, et al. Stereotactic radiosurgery for patients with multiple brain metastases (JLGK0901): a multi-institutional prospective observational study. Lancet Oncol. 2014;15(4):387-95.

14. Abbott AM, Dossett LA, Loftus L, Sun W, Fulp W, Sokol GH, et al. Intraoperative radiotherapy for early breast cancer and age: clinical characteristics and outcomes. Am J Surg. 2015;210(4):624-8.

15. Rothschild SI, Thommen DS, Moersig W, Muller P, Zippelius A. Cancer immunology - development of novel anti-cancer therapies. Swiss Med Wkly. 2015;145:w14066 Summarises the mechanism of action and subsequent clinical studies of immune checkpoint antibodies in oncology with a particular focus on melanoma and lung cancer.

16. Zhang J, Yang PL, Gray NS. Targeting cancer with small molecule kinase inhibitors. Nat Rev Cancer. 2009;9:28-39.

17. Sharma PS, Sharma R, Tyagi T. VEGF/VEGFR pathway inhibitors as anti-angiogenic agents: present and future. Curr Cancer Drug Targets. 2011;11(5):624-53.

18. Verheul HM, Pinedo HM. Possible molecular mechanisms involved in the toxicity of angiogenesis inhibition. Nat Rev Cancer. 2007;7(6):475-85.

19. Wozel G, Sticherling M, Schon MP. Cutaneous side effects of inhibition of VEGF signal transduction. Journal der Deutschen Dermatologischen Gesellschaft $=\mathrm{J}$ German Soc Dermatol : JDDG. 2010;8(4):243-9.

20.• Bodnar RJ. Anti-angiogenic drugs: involvement in cutaneous side effects and wound-healing complication. Adv Wound Care. 2014;3(10):635-46 Awareness is needed when treating patients on anti-angiogenic drugs so as not to exacerbate potential wound-healing complications when performing surgical procedures. When performing a surgical procedure, the impact ofadverse effects from the use of anti-angiogenic drugs should be considered to ensure the welfare of the patient.

21. Chida K, Nakanishi K, Shomura H, Homma S, Hattori A, Kazui K, et al. Spontaneous regression of transverse colon cancer: a case report. Surg Case Reports. 2017;3(1):65.
22. Challis GB, Stam HJ. The spontaneous regression of cancer: a review of cases from 1900 to 1987. Acta Oncol. 1990;29(5):54550 .

23.• Ascierto PA, Brugarolas J, Buonaguro L, et al. Perspectives in immunotherapy: meeting report from the Immunotherapy Bridge (2930 November, 2017, Naples, Italy). J Immunother Cancer. 2018;6: 69 Immunotherapy represents the third important wave in the history of the systemic treatment of cancer after chemotherapy and targeted therapy and is now established as a potent and effective treatment option across several cancer types. With over 3000 immuno-oncology trials ongoing, involving hundreds of research institutes across the globe. The potential use of these different immunotherapeutic options in various combinations with one another and with other treatment modalities is an area of particular promise.

24. Hansel TT, Kropshofer H, Singer T, Mitchell JA, George AJT. The safety and side effects of monoclonal antibodies. Nat Rev Drug Discov. 2010;9:325-38.

25. Weiner LM, Dhodapkar MV, Ferrone S. Monoclonal antibodies for cancer immunotherapy. Lancet. 2009;373(9668):1033-40.

26. Major EO. Progressive multifocal leukoencephalopathy in patients on immunomodulatory therapies. Annu Rev Med. 2010;61:35-47.

27. Kounis NG, Zavras GM. Histamine-induced coronary artery spasm: the concept of allergic angina. British J Clin Practice. 1991;45(2):121-8.

28. Kounis NG, Soufras GD, Tsigkas G, Hahalis G. Adverse cardiac events to monoclonal antibodies used for cancer therapy: the risk of Kounis syndrome. Oncoimmunology. 2014;3:e27987.

29. Coles AJ, Wing M, Smith S, Coraddu F, Greer S, Taylor C, et al. Pulsed monoclonal antibody treatment and autoimmune thyroid disease in multiple sclerosis. Lancet. 1999;354(9191):1691-5.

30. Guglin M, Cutro R, Mishkin JD. Trastuzumab-induced cardiomyopathy. J Card Fail. 2008;14(5):437-44.

31. La-Beck NM, Jean GW, Huynh C, Alzghari SK, Lowe DB. Immune checkpoint inhibitors: new insights and current place in cancer therapy. Pharmacotherapy. 2015;35(10):963-76.

32. Topalian SL, Drake CG, Pardoll DM. Immune checkpoint blockade: a common denominator approach to cancer therapy. Cancer Cell. 2015;27(4):450-61.

33. Corsello SM, Barnabei A, Marchetti P, De Vecchis L, Salvatori R, Torino F. Endocrine side effects induced by immune checkpoint inhibitors. J Clin Endocrinol Metab. 2013;98(4):1361-75.

34. Lee DW, Gardner R, Porter DL, Louis CU, Ahmed N, Jensen M, et al. Current concepts in the diagnosis and management of cytokine release syndrome. Blood. 2014;124(2):188-95.

35. Bilusic M, Madan RA. Therapeutic cancer vaccines: the latest advancement in targeted therapy. Am J Ther. 2012;19(6):e172-81.

36. Lee HJ, Kim YA, Sim CK, Heo SH, Song IH, Park HS, et al. Expansion of tumor-infiltrating lymphocytes and their potential for application as adoptive cell transfer therapy in human breast cancer. Oncotarget. 2017;8(69):113345-59.

37.• Yang L, Yu H, Dong S, Zhong Y, Hu S. Recognizing and managing on toxicities in cancer immunotherapy. Tumor Biol. 2017;39(3): 1010428317694542 Treatment toxicity spectrums vary greatly even in same type of cancers. Some side effects are reversible, and can be processed through the standard medicines. However, serious toxicities are lethal, which should be frequently followed-up and identified at an early stage. 